\title{
The effects of dietary restriction and exercise on the volume of adipocytes in two intra-orbital depots in the guinea-pig
}

\author{
By CHRISTINE A. MATTACKS AND CAROLINE M. POND* \\ Department of Biology, The Open University, Walton Hall, Milton Keynes MK7 6AA
}

(Received 3 July 1984-Accepted 12 October 1984)

\begin{abstract}
1. The volume of adipocytes in two intra-orbital sites and fourteen superficial and intra-abdominal sites, and the total adipocyte complement have been measured in virgin and reproductive guinea-pigs maintained on several different regimens of diet and exercise.

2. The adipocytes around the ocular muscles at the back of the orbit (peripheral fat) are always larger than those just behind the eyeball (orbital fat).

3. The adipocytes in both the intra-orbital sites are significantly larger in guinea-pigs whose total adipocyte complement is smaller than one standard deviation from the mean, than in those which have a normal-size or large adipocyte complement.

4. The volume of intra-orbital adipocytes correlates very significantly with the volume of adipocytes in superficial and intra-abdominal sites in guinea-pigs which have large adipocyte complements, correlates weakly in those with normal adipocyte complements and not at all in those with small adipocyte complements.

5. It is suggested that there may be fewer intra-orbital adipocytes in animals which have small adipocyte complements, and that, because the intra-orbital adipose tissue occupies a constant volume, the adipocytes in these sites become larger when they are less numerous.
\end{abstract}

Pond et al. (1984b) studied the volume of adipocytes in precisely defined sites in intermuscular adipose depots of guinea-pigs maintained on different regimens of diet and exercise. They showed that, particularly in animals which exercised or fasted regularly, proximity to the same or functionally synergistic muscles was one factor which promoted a higher correlation between the volumes of adipocytes in different sites. The volume of these adipocytes was determined in part by local, site-specific factors, acting in conjunction with the neural and endocrinological mechanisms which regulate adipocyte volume in relation to the energy balance of the animal as a whole. It was suggested that the effect was due to mechanical forces exerted by actively contracting muscles on adipocytes in adjacent intermuscular sites. In the present paper we report evidence that non-muscular mechanical constraints can also play a role in determining the volume of adipocytes.

The adipose tissue inside the mammalian eyesocket (orbit) resembles intermuscular adipose tissue in that the gross dimensions of the whole depot are limited by the mechanical properties of the surrounding tissues. As Warwick (1976) put it, 'the orbital fat (of the human eye) compactly fills all the space not occupied by the other structures'. The eyeball, the optic nerve and the ocular muscles do not normally undergo significant changes in volume and they, together with the intra-orbital adipose tissue, are almost completely enclosed in an indeformable bony capsule and tough sclera. The intra-orbital adipose depot is therefore unusual in that its total volume probably remains almost constant during adult life. It is therefore a very suitable system on which to study the contribution of adipocyte enlargement and adipocyte proliferation to filling an anatomical space of fixed volume. 


\section{MATERIALS AND METHODS}

The fifty-three guinea-pigs used included twenty-four males and twenty-nine females, thirty Dunkin Hartleys, sixteen Bolivians and seven Abyssinians. They were at least 15 weeks old when the special regimens of diet and exercise started, and at least 20 weeks old when killed. The animal husbandry, tissue storage, adipocyte sizing techniques and three of the regimens of diet and exercise were the same as those described by Pond et al. (1984a); many of the guinea-pigs used for that work were also used for the present study. The three groups studied were: sedentary, ad lib.-fed guinea-pigs kept indoors in small cages; animals given a restricted diet and kept indoors in small cages; animals kept in an outdoor pen in which the food and the water were placed $6 \mathrm{~m}$ apart. The restricted-diet guinea-pigs studied by Pond et al. (1984a) had significatly fewer adipocytes than either of the groups which were ad lib.-fed.

In the hope of finding animals with very large adipocyte complements, we also examined some older males and females from the breeding stock; all these guinea-pigs were maintained in standard laboratory cages of $0.5 \mathrm{~m}^{2}$ throughout their lives. The sedentary, ad lib.-fed group therefore includes some older, ex-breeding males. We also examined two groups of post partum, non-pregnant females. The ten 'fat mothers' were allowed to feed ad $l i b$. for at least 1 month after weaning a litter. Six females, which had raised an average of twelve offspring each (range seven to twenty) in two to five litters, were either killed just after they had weaned a litter or placed on the restricted diet regimen for 9-11 weeks. When killed, these restricted-diet mothers weighed 15-28\% less than their peak non-pregnant weight and were much older at death than the similarly treated virgins (see Table 1, p. 209). A sixth group were kept under the same conditions as the exercising animals, then transferred for the last 6-8 weeks of their lives to a smaller enclosure ( $2 \mathrm{~m}^{2}$ in area) where food and water were adjacent and available $a d l i b$.

The animals were killed with an overdose of urethane $(6-10 \mathrm{ml}$ of a $500 \mathrm{mg} / \mathrm{ml}$ solution injected intraperitoneally). The intact body was left to cool overnight in a refrigerator and dissected completely, as described by Pond et al. (1984a). The entire contents of both orbital cavities were either removed at the time the rest of the carcass was dissected or the whole head stored at -15 to $-17^{\circ}$ for up to 3 months.

Warwick (1976) distinguished two adipose masses in the eyesocket of humans: the inner 'orbital' fat mass immediately behind the eyeball and around the optic nerve, and 'peripheral' fat between the recti ocular muscles and the periorbital membranes. In humans, and in guinea-pigs, this latter fat mass is thickest at the back of the orbit, near the point of insertion of the ocular muscles. We studied two adipose tissue samples from each eye: one (NREYE) was taken from just behind the eyeball, close to the point at which the optic nerve emerges, and the other (NRBM) was taken from the superficial side of the ocular muscles near to their attachment to the bone at the back of the orbit.

The total mass of adipose tissue in each orbit rarely exceeds $100 \mathrm{mg}$ and the small groups of adipocytes have a great deal of connective, neural and vascular tissue associated with them. It is almost impossible to dissect out all the adipose tissue in order to weigh it accurately enough to provide a basis for reliable estimation of the tissue cellularity. The total number of adipocytes in each orbit is approximately $2 \times 10^{5}$, or about $0.2 \%$ of the total adipocyte complement of the body.

The sample of adipose tissue was covered with a drop of phosphate-buffered saline $(9 \mathrm{~g}$ sodium chloride/1) and the diameter of forty adipocytes from each sample was measured immediately after dissection, in the way described by Pond et al. (1984a). The mean adipocyte volume for each sample was calculated using the equation developed by Goldrick (1967). The mean volume of adipocytes in the fourteen superficial and intra-abdominal 
Table 1. Age and body composition of guinea-pigs in the six treatment groups

(Mean values and standard deviations)

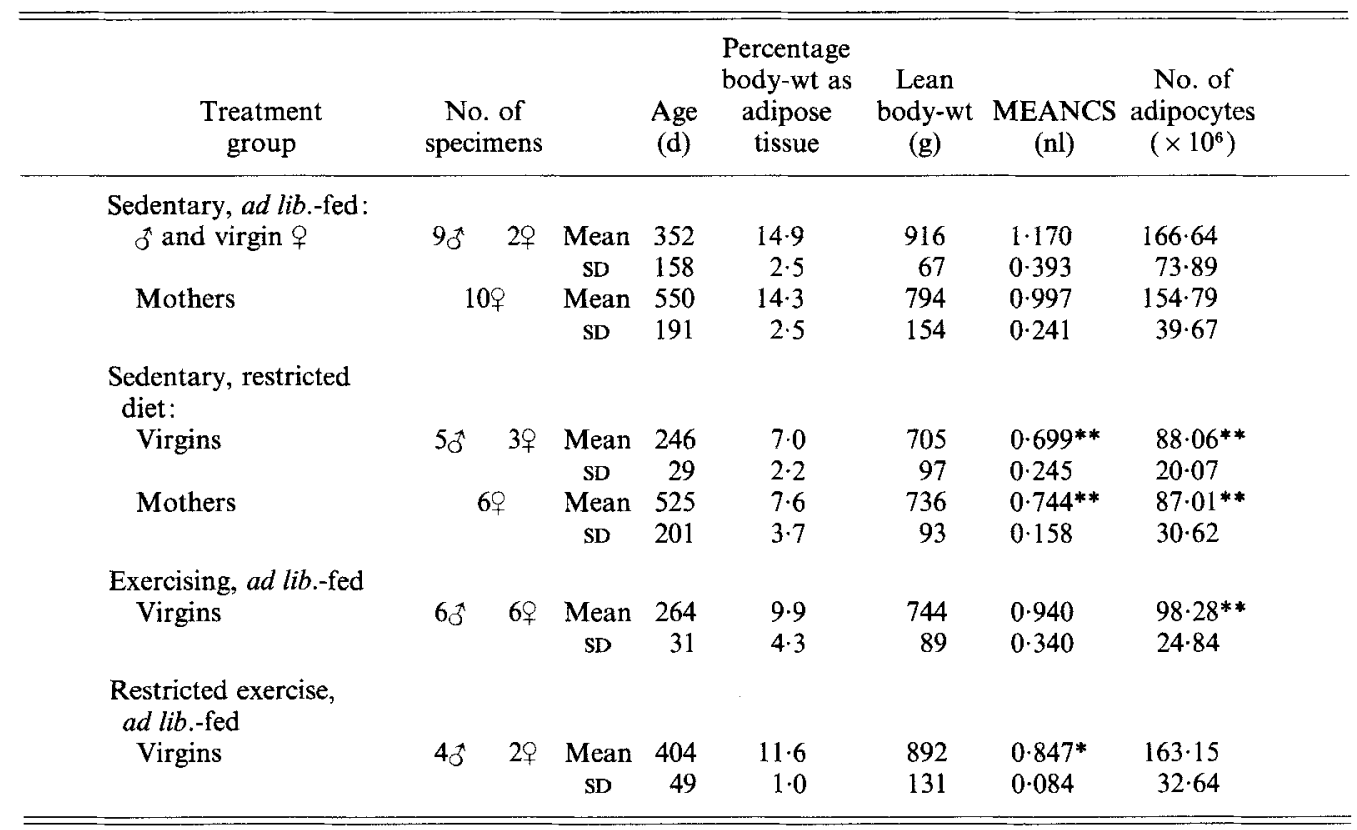

MEANCS (mean cell size), index of the average volume of adipocytes in the body as a whole, calculated as explained on p. 208 and in Pond et al. (1984a).

Values are significantly different from those for sedentary, ad lib.fed males and virgin females: ${ }^{*} P<0.05$, ** $P<0.01$. All other differences in MEANCS and number of adipocytes are not significantly different $(P>0 \cdot 05)$.

adipose depots studied by Pond et al. (1984a) was also measured for each guinea-pig. Both the index of the average volume of adipocytes in the body as a whole (MEANCS, mean cell size) and the total adipocyte complement were based on measurements of the mean volume of adipocytes in sixteen superficial, intra-abdominal and intermuscular sites and were determined as described in Pond et al. $(1984 a, b)$. The results were analysed using conventional computing techniques and Statistical Package for the Social Sciences (Nie et al. 1975).

\section{RESULTS}

Table 1 shows the mean age and aspects of the body composition of the six groups of guinea-pigs studied. As we hoped, many of the older breeding-stock guinea-pigs had substantially larger adipocyte complements than the younger, virgin animals maintained on regimens of diet and exercise similar to those regimens that we studied previously (Pond et al. 1984a). The ex-breeding females maintained on the restricted diet also had significantly fewer adipocytes than reproductive females of the same age which had been fed ad lib. (Student's $t$ test: $t 3.83, P<0.01,14 \mathrm{df}$ ), although there was no significant difference in the average lean body mass of the two groups (Student's $t$ test: $t 14 \mathrm{df}$ ).

The volumes of adipocytes from homologous sites in the left and right eyes were very similar in all animals, and the two sets of values correlated closely. For all groups the volume of adipocytes in the NREYE site correlated significantly with that of adipocytes in the 


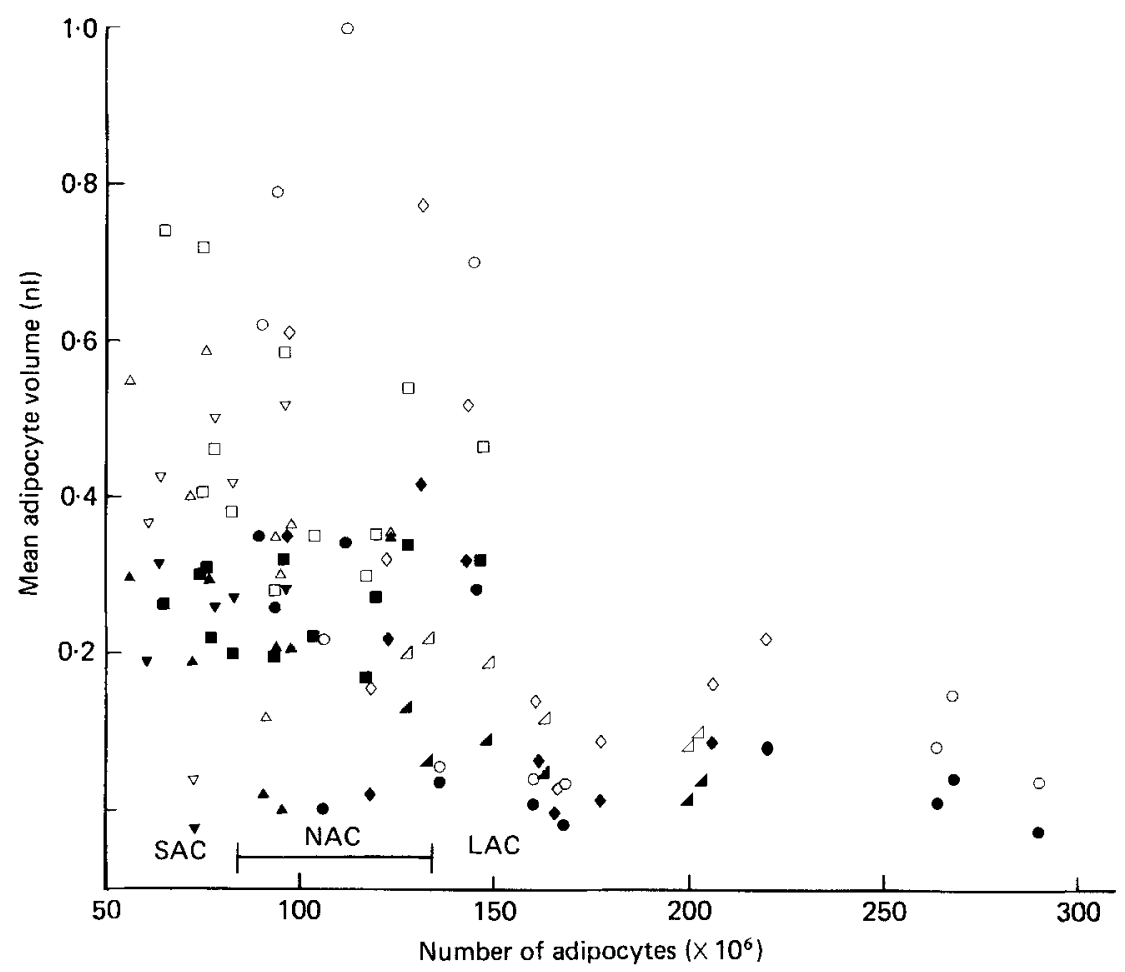

Fig. 1. The relation between total adipocyte complement $\left(\times 10^{6}\right)$ and the mean volume (nl) of adipocytes behind the eyeball (NREYE) $(\bullet \Delta \nabla \square \Delta)$ and of adipocytes between the ocular muscles (NRMB) $(\bigcirc \diamond \Delta \nabla \square \Delta) .(\odot, \bigcirc)$ Sedentary, ad lib.-fed males and virgin females; $(\diamond, \diamond)$ sedentary, ad lib.-fed mothers; $(\mathbf{A}, \triangle)$ sedentary, restricted-diet virgins; $(\nabla, \nabla)$ sedentary, restricted-diet mothers; $(\square, \square)$ exercising, ad lib.-fed virgins; $(\boldsymbol{\Lambda}, \Delta)$ restricted exercise, ad lib.-fed virgins. SAC, small adipocyte complement; NAC, normal adipocyte complement; LAC, large adipocyte complement. The mean adipocyte complement for ad lib.-fed virgin guinea-pigs aged about 9 months was $108.64 \times 10^{6}$ (SD $25.3 \times 10^{6}$ ). Specimens for which the adipocyte complement falls more than 1 SD from this average value were placed in SAC or LAC.

NRMB site $(r 0.662-0.945, P<0.01)$. The mean of the samples from the left and right eyes was used for the rest of the analysis.

Fig. 1 shows the mean volumes of adipocytes in NREYE and NRMB as a function of the total number of adipocytes in the body. In every case the adipocytes in the peripheral adipose tissue at the back of the eyesocket (NRMB) were larger than those behind the eyeball (NREYE). The measurements were scattered but there was a clear inverse relation between adipocyte volume and total adipocyte complement for both sites (NREYE: $r-0.545, P<0.01$; NRMB: $r-0.513, P<0.01)$. In other words, the largest intra-orbital adipocytes were found in the guinea-pigs which had the fewest adipocytes in the rest of the body.

In Table 2, the measurements have been divided into three categories according to the size of their total adipocyte complement in relation to the mean adipocyte complement reported for young virgin guinea-pigs by Pond et al. (1984a). They found that the mean adipocyte complement for both sexes of sedentary ad lib.-fed guinea-pigs was $108.64 \times 10^{6}$ (SD $25 \cdot 3 \times 10^{6}$ ) cells. The thirteen guinea-pigs designated as small adipocyte complement (SAC) had fewer than $83.34 \times 10^{6}$ adipocytes. As can be seen from Fig. 1, all these guinea-pigs came from the restricted-diet or exercised groups. Those in the normal adipocyte complement 
Table 2. Number of adipocytes, lean body-weight and mean volume of adipocytes behind the eyeball $(N R E Y E)$ and around the ocular muscles $(N R M B)$ in fifty-three guinea-pigs divided according to total adipocyte complement. The mean adipocyte complement for young virgin ad lib.-fed guinea-pigs was $108.64 \times 10^{6}\left(\mathrm{SD} 25 \cdot 3 \times 10^{6}\right)$ adipocytes

(Mean values and standard deviations)

\begin{tabular}{|c|c|c|c|c|c|c|}
\hline & \multicolumn{2}{|c|}{$\begin{array}{l}\text { Small adipocyte } \\
\text { complement (n 13) }\end{array}$} & \multicolumn{2}{|c|}{$\begin{array}{l}\text { Normal adipocyte } \\
\text { complement }(n 22)\end{array}$} & \multicolumn{2}{|c|}{$\begin{array}{l}\text { Large adipocyte } \\
\text { complement ( } n 18)\end{array}$} \\
\hline & Mean & SD & Mean & SD & Mean & SD \\
\hline No. of adipocytes $\times 10^{8}$ & $72 \cdot 02^{* *}$ & $8 \cdot 23$ & $108 \cdot 43$ & $14 \cdot 75$ & $187 \cdot 49 * *$ & $46 \cdot 80$ \\
\hline Lean body-wt $(g)$ & 722 & 114 & 762 & 113 & $899 * *$ & 97 \\
\hline $\operatorname{MEANCS}(\mathrm{nl})$ & $0.774 *$ & 0.261 & $1 \cdot 045$ & 0.401 & 0.900 & 0.164 \\
\hline NREYE (nl) & 0.437 & 0.086 & 0.423 & $0 \cdot 130$ & $0 \cdot 286^{* *}$ & 0.114 \\
\hline NRMB (nl) & 0.657 & $0 \cdot 180$ & 0.627 & $0 \cdot 241$ & $0.377 * *$ & $0 \cdot 193$ \\
\hline
\end{tabular}

MEANCS (mean cell size), index of the average volume of adipocytes in the body as a whole, calculated from sixteen body sites as explained on p. 208 and in Pond et al. (1984a).

Values are significantly different from those for normal adipocyte complement group: $P<0 \cdot 05, * * P<0 \cdot 0$. All other differences are not significantly different $(P>0.05)$.

(NAC) category had a total number of adipocytes which were within 1 SD of the mean adipocyte complement reported previously. The eighteen animals in the large adipocyte complement (LAC) group had adipocyte complements greater than $134 \times 10^{6}$ cells. This category included animals from the two sedentary ad lib.-fed groups and some from the food-restricted, exercised group (see Fig. 1). In our sample, there was no significant difference in the lean-body mass of SAC and NAC groups (Student's $t$ test, $t 1 \cdot 006,33 \mathrm{df}$ ). There was also no significant difference between the NAC and LAC groups in the index of mean adipocyte volume for the whole animal (MEANCS) but, as expected, MEANCS was significantly smaller in the SAC group than in the NAC group (Student's $t$ test: $t 2.42$, $P<0.05,33 \mathrm{df}$ ); however, the MEANCS of the SAC group was not significantly different from that of the LAC group (Student's $t$ test: $t 1 \cdot 54,29 \mathrm{df}$ ).

For both NREYE and NRMB, adipocytes from the SAC group were significantly larger than those in the same sites in the LAC animals (Student's $t$ test, NREYE: $t 4 \cdot 203, P<0.01$, $29 \mathrm{df}$; NRMB: $t 4 \cdot 146, P<0 \cdot 01,29 \mathrm{df}$ ). The volumes of adipocytes from the LAC group also differed significantly from those in the NAC category (Student's $t$ test, NREYE: $t 3.549$, $P<0.01,31 \mathrm{df}$; NRMB: $t 3.643, P<0.01,33 \mathrm{df}$ ); the differences between the SAC group and the NAC group were not significant at $P<0.05$. The mean volume of adipocytes in the peripheral fat (NRMB) in the orbit of the SAC group was not significantly different from MEANCS of the same animals (Student's $t$ test: $t 1 \cdot 331$, df 24), although in the other two groups the volumes of adipocytes from both intra-orbital sites were clearly very much smaller than the MEANCS.

For the LAC guinea-pigs, most of the correlations between the mean volumes of adipocytes in both NREYE and NRMB and the mean volumes of adipocytes in the fourteen superficial and intra-abdominal sites studied by Pond et al. (1984a) were significant ( $r 0.357-0.852$, all but three significant at $P<0.05,16 \mathrm{df}$ ). In the case of the NAC guineapigs, the volume of adipocytes at the NREYE site did not correlate significantly with that of adipocytes in any of the other adipose depots studied by Pond et al. $(1984 a, b)(r 0 \cdot 420-0.095$, $20 \mathrm{df}$. The volume of adipocytes in the NRMB site correlated significantly with the volume of those elsewhere in the body, but the correlations were much weaker $(r 0.675-0.496$, 
$P<0.05,20 \mathrm{df})$ than those between the volumes of adipocytes in different adipose depots reported previously (Pond et al. 1984a, b). In the SAC group, no significant correlation could be demonstrated between either of the two intra-orbital sites and any of the superficial or intra-abdominal sites studied previously $(r 0 \cdot 183-0 \cdot 017,11 \mathrm{df})$.

\section{DISCUSSION}

The similarity between the volume of adipocytes from the two eyes was expected from the work of Pond et al. (1984a) who reported a high and constant similarity between the volume of adipocytes from left-right homologous pairs of sites. It also confirms the suggestion made by Warwick (1976) that the gross dimensions of the intra-orbital adipose tissue must be identical in the two eyes, if coordinated eye movements and binocular vision is to be possible.

Some other aspects of the present findings suggest an apparent paradox: the thinnest guinea-pigs, which had the smallest and the fewest adipocytes elsewhere in the body, had the largest intra-orbital adipocytes. The closest correlations between the volumes of the intra-orbital adipocytes and those in adipose depots elsewhere in the body are found in animals with the largest adipocyte complements, most of which were sedentary and fed ad lib. This result is the opposite expected from the work of Pond et al. (1984a), who found that the correlations between the volume of adipocytes in superficial, intra-abdominal and intermuscular sites were significantly higher in the restricted-diet and exercised guinea-pigs than in the sedentary ad lib.-fed animals.

Pond et al. (1984a) found that there were fewer adipocytes in all the adipose depots studied in the restricted-diet animals than in the two groups of ad lib.-fed guinea-pigs, and that the reduction in cellularity was greatest in those sites which normally comprised relatively small adipocytes. It is possible that the number of adipocytes in the eye socket is also lower in the thinner guinea-pigs. The space inside the eye socket was not measured, but it is most unlikely that it is smaller in the thinner guinea-pigs. None of the treatments were begun until the guinea-pigs were at least 15 weeks old, at which time the eyes and skull would be almost fully grown. The eyes and the skull are relatively large at birth and continue to grow rapidly during sucking, but the rate of growth of the skull slows down at a much earlier age than that of the rest of the body (Hughes \& Tanner, 1970). There was also no significant difference in the lean-body mass of the SAC and NAC animals (see Table 2).

We suggest that the intra-orbital adipocytes expand to fill the constant space available for adipose tissue in the orbit; when fewer adipocytes are present at these sites, those present enlarge so that the total volume of the depot is unchanged. Enlargement of intra-orbital adipocytes takes place even though the volume of most of the adipocytes elsewhere in the body may be undergoing a net reduction in volume at the same time; as a result, there is no significant correlation between the volume of intra-orbital adipocytes and those elsewhere in the body of guinea-pigs with normal or small adipocyte complements. The volumes of intra-orbital adipocytes of guinea-pigs with large adipocyte complements correlate closely with the volume of those in other sites; this observation suggests that the development of intra-orbital adipocytes of volumes which correspond to the volume of adipocytes in superficial and intra-abdominal sites elsewhere in the same animal is promoted by unimpeded proliferation (or recruitment) of adipocytes. These findings support the concept, suggested by Pond et al. (1984b) in connection with adipocytes in the popliteal fat mass, that mechanical constraints imposed by the tissues surrounding adipocytes can play a part in determining their volume. It is possible that similar mechanical effects may also be important in determining the volume of adipocytes in other adipose depots, particularly those of elderly humans, whose skin, mesenteries and fascia have become less deformable 
with age and hence less able to accommodate changes in the total volume of associated adipose tissues.

Aronovsky et al. (1963) compared the response to fasting and the uptake of isotopically labelled fatty acids of intra-orbital, 'subcutaneous' (ventral groin (GV) site (Pond et al. $1984 a)$ ) and omental adipose tissue from fasted and fed cats. They found that in the orbital adipose tissue the release and incorporation of fatty acids was measurable, but much slower and less extensive than in the other sites in vitro. During fasting, net lipid mobilization in the orbital adipocytes actually decreased slightly, while that of their omental and groin samples increased. Aronovsky et al. (1963) could not explain their observations; we propose that intra-orbital adipocytes can take up fatty acids from the blood under endocrinological conditions which promote release of lipid from adipocytes in most of the other adipose depots which have been studied. We suggest that the biochemical basis for this effect warrants further study.

The present study has demonstrated that the intra-orbital adipocytes can undergo changes in volume which are quantitatively and qualitatively quite different from those undergone at the same time by adipocytes in depots elsewhere in the body. When the total adipocyte complement is smaller, the overall volume of the intra-orbital adipose depot does not necessarily diminish because the remaining adipocytes enlarge. The findings are consistent with the suggestion that mechanical constraints imposed by the physical dimensions of the anatomical space available to intra-orbital adipose tissue can play a role in determining adipocyte volume.

The authors thank Dawn Sadler for skilled husbandry of the experimental animals and Marion Thompson for help with some of the dissections.

\section{REFERENCES}

Aronovsky, E., Levari, R., Kornbleuth, W. \& Wertheimer, E. (1963). Investigative Ophthalmology 2, $259-264$. Goldrick, R. B. (1967). American Journal of Physiology 212, 777-782.

Hughes, P. C. R. \& Tanner, J. M. (1970). Journal of Anatomy 106, 349-370.

Nie, N. H., Hull, C. H., Jenkins, J. G., Steinbrenner, K. \& Brent, D. H. (1975). Statistical Package for the Social Sciences, 2nd ed. New York: McGraw-Hill.

Pond, C. M., Mattacks, C. A. \& Sadler, D. (1984a). British Journal of Nutrition 51, 415-424.

Pond, C. M., Mattacks, C. A. \& Sadler, D. (1984b). British Journal of Nutrition 51, 425-433.

Warwick, R. (1976). Anatomy of the Eye and Orbit, 7 th ed. London: H. K. Lewis \& Co. 